\title{
SOCIEDAD DE LA GEOINFORMACIÓN Y CONDUCTA ESPACIAL DEL CIUDADANO COMO NUEVOS DESA- FÍOS PARA LA GEOGRAFÍA
}

\author{
Antonio MORENO JIMÉNEZ (antonio.moreno@uam.es) \\ Departamento de Geografía. Universidad Autónoma de Madrid
}

Recibido: $15 / 02 / 2015$

Aceptado: 15/07/2015

RESUMEN: Las innovaciones que caracterizan a la denominada sociedad de la geoinformación (SGI) y a su tecnosfera han permitido desarrollar un poderoso ciberespacio, cuyo acceso, cada vez más general entre la ciudadanía, está cambiando intensamente la tradicional interacción hombre-espacio, al condicionar tanto la formación de decisiones individuales, como las conductas espaciales, fenómenos ambos que requieren una atención preferente de nuestra disciplina. Partiendo de una serie de conceptos geográficos importantes sobre el particular y de una breve caracterización de la SGI, en este artículo se pretende destacar y reflexionar sobre algunas consecuencias novedosas observables en las búsquedas de información en el ciberespacio como apoyo a la movilidad espacial de los individuos. El sentido y meta últimos de ello estriba en explorar oportunidades emergentes para resituar y reforzar el valor del conocimiento geográfico para el ciudadano en este contexto.

$\boldsymbol{P A L A B R} \boldsymbol{A S C L A V E}$ : sociedad de la geoinformación, ciberespacio, entorno personal próximo, movilidad espacial, geotecnologías.

\section{GEOINFORMATION SOCIETY AND CITIZEN'S SPATIAL BEHAVIOR AS NEW CHALLENGES FOR GEOGRAPHY}

ABSTRACT: Innovations underlying the so-called geoinformation society (GIS) and its «technosphere» have allowed developing a powerful cyberspace. The expanding access to it by citizenship is strongly changing the traditional man-space interaction, because that conditions individual decision making and spatial behavior, a couple of processes requiring priority attention in our science. Based on a set of important geographical concepts about this field and on some features of GIS, in this paper it is intended to emphasize and reflect on some new consequences observed when individuals carry out information search in cyberspace to support their mobility across space. The ultimate intended goal lies in exploring the emerging opportunities for repositioning and strengthening the value of geographical knowledge for citizenship in this context.

KEY WORDS: geoinformation society, cyberspace, proximal personal milieu, spatial mobility, geotechnologies. 


\section{INTRODUCCIÓN}

Como adujo MUMFORD (1934) en su conocida obra Técnica y civilización, a lo largo de la historia las condiciones técnicas han marcado la existencia de la humanidad de manera tan decisiva, que definieron etapas con rasgos diferenciales según los progresos en dichas técnicas. Aunque por su antigüedad el autor no pudo conocer nuestro mundo presente, no son pocos los estudiosos que han establecido con meridiana claridad que desde hace varias décadas las innovaciones tecnológicas nos han llevado a una fase nueva y sustancialmente distinta de las descritas por aquél autor. Sociedad de la información y de la comunicación, sociedad informacional, sociedad del conocimiento son expresiones que se hallan bien extendidas en la bibliografía y forman parte ya del acervo común para referirse a esta nueva etapa. Obviando la glosa de tales conceptos, por sabidos, resulta a nuestros fines conveniente reutilizar aquí la expresión de «sociedad de la información geográfica» o de la geoinformación (SGI) que ya se ha manejado con anterioridad (vid. MORENO, 2010; 2013) y que pone el énfasis en la presencia masiva de geodatos digitales y de los medios electrónicos para su obtención, almacenamiento, distribución y uso habitual por la sociedad.

El retraso casi hasta el siglo actual con que el componente espacial de los datos ha logrado una presencia y generalización en la masa de información digital que nos acompaña apenas ha permitido tomar conciencia todavía de las trascendentales mutaciones que está ocasionando y que cabe calificar de revolucionarias, y no como simple evolución, por cuanto están alterando radicalmente las bases sobre las que nuestra existencia discurre y produciendo cambios cuantitativos y cualitativos sin precedentes (vid. WILSON et al., 2013). El fenómeno, aún en su fase incipiente a nuestro juicio (vid. MORENO, 2004), está exhibiendo las etapas típicas de la difusión de innovaciones, en las que ciertos lugares y agentes (individuales y colectivos u organizaciones) son pioneros, en tanto que otros van rezagados; sin embargo, permite ya sustentar algunas reflexiones generales sobre el mismo, por cuanto hay suficientes manifestaciones como para reconocer los cambios, valorar la enjundia de los mismos e, incluso, avistar horizontes próximos con bastante verosimilitud.

Al respecto, uno de los frentes en el que las transformaciones están siendo más intensas concierne a las posibilidades que la SGI ofrece a los ciudadanos al acceder al ciberespacio, a su movilidad en ese ámbito y a cómo ambos factores repercuten y se integran en los desplazamientos de las personas (vid. JANELLE y HODGE, 2000; THILL, 2009). En suma, se trata de cambios que se van plasmando en una creciente movilidad tanto en el espacio virtual o digital, como en el físico, lo que demanda revisar, a la luz del nuevo contexto, algunos conceptos geográficos fundamentales que se acuñaron hace un tiempo para 
analizar y entender dicha movilidad. Esos fenómenos incipientes suponen también oportunidades y retos para las comunidades científicas y profesionales de los geógrafos que conviene explorar.

De acuerdo con ello, en este ensayo nos proponemos realizar un conjunto de consideraciones sobre este proceso en curso, por cuanto la trascendencia para la humanidad es tal que la disciplina geográfica y sus cultivadores no pueden eludir la obligación de recapacitar sobre su papel y agenda de trabajo, el cual habría de producir y diseminar conocimiento que contribuya relevantemente a las metas socialmente compartidas. El objetivo no es otro que el de estimular el debate crítico y el reposicionamiento de nuestro quehacer en este nuevo horizonte temporal, por cuanto ello resulta urgente y necesario, no solo para nuestro colectivo académico y profesional, sino también para la sociedad.

A tal fin, en el siguiente apartado se recuerdan algunos conceptos geográficos clásicos sobre la movilidad espacial y se añade alguno nuevo como el de «entorno personal próximo», que entendemos ofrece sugerentes posibilidades para el análisis en el presente contexto. A continuación se subrayan brevemente algunas características importantes de la sociedad de la geoinformación (SGI) y del ciberespacio, como nuevos marcos de la existencia personal, para luego exponer ciertas consecuencias de la SGI en el conocimiento y conducta espacial del ciudadano. El siguiente apartado aborda algunos retos para la geografia en la SGI, particularmente los atingentes a oportunidades y demandas suscitadas por las prácticas espaciales del ciudadano que emergen en este marco vital, para finalizar con unas breves conclusiones.

\section{ALGUNOS CONCEPTOS RELEVANTES SOBRE LA CON- DUCTA ESPACIAL DEL INDIVIDUO}

Siendo, al decir de Kant, el espacio, junto con el tiempo, las formas de la sensibilidad o intuiciones puras del hombre que confieren su estructura a las cosas que conocemos, su papel en la conformación de la existencia humana resulta esencialmente determinante, por cuanto nos permite su representación y su utilización derivada. La espacialidad de los seres se constituye en el basamento para el despliegue de la vida, al establecer la disposición mental de esa representación de la realidad y posibilitar así su aprovechamiento por el ser humano.

En los numerosos estudios sobre geografía de la percepción y de la conducta espacial humana se han construido algunos conceptos imprescindibles (vid. por ejemplo CAPEL, 1973; GOLLEDGE y STIMSON, 1997; AMEDEO et al. 2009), que conviene rememorar sucintamente aquí, tales como mapa mental, alusivo a la representación mental del espacio conocido por el sujeto y en el 
cual se apoya su patrón comportamental, campo de información espacial, CIE (information / cognitive field), como ámbito sobre el que el individuo posee conocimiento por algún medio directo o indirecto, y campo de actividad o interacción espacial, CAE (action / activity field), como el conjunto de lugares con los que el individuo mantiene contactos como resultado de sus actividades frecuentes (vid. FIGURA 1). Su relevancia es sobradamente conocida en parcelas como la geografía del transporte (e.g. POTRYKOWSKI y TAYLOR, 1984) o la del comercio y servicios (e.g. TIMMERMANS, 2004). La relación entre ellos está también bien sustentada, operando los dos primeros como alimentadores y condicionantes del tercero; es decir, el individuo construye sus decisiones espaciales sobre la base del stock de datos disponibles en su mapa cognitivo acerca de los entes y posibilidades localizadas.

Figura 1. El campo de actividad espacial (CAE) de un individuo

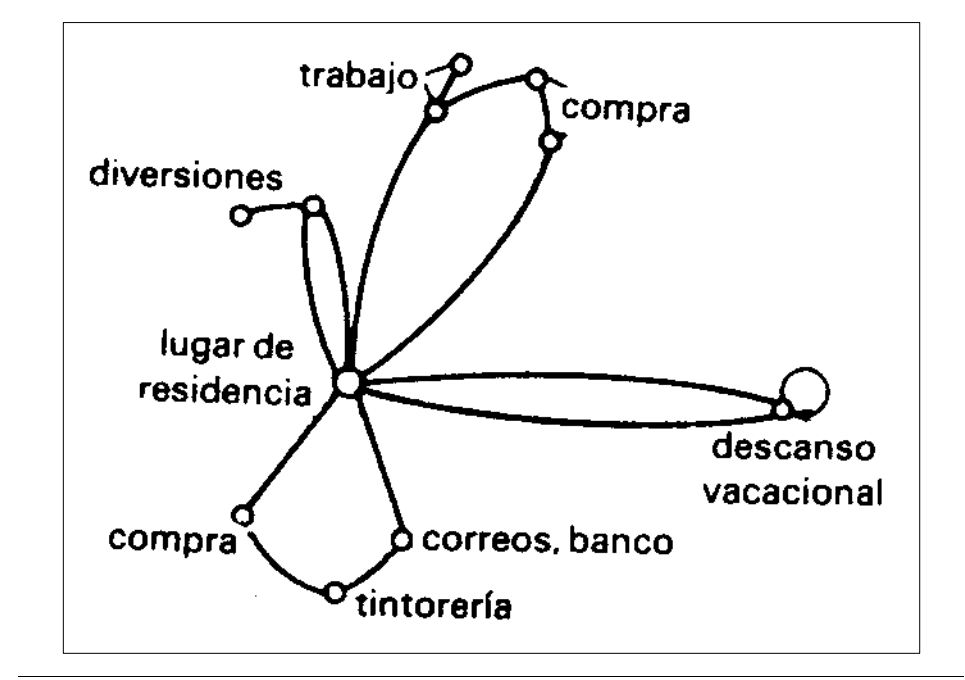

FUENTE.: POTRYKOWSKI y TAYLOR (1984: 279)

A la luz de las constataciones empíricas y de la interpretación de diversos estudios cabría postular axiomáticamente la existencia de dos tipos de impulsos informativos sobre fenómenos (entes, procesos o atributos) espaciales que a menudo operan de manera convergente:

a) Por un lado, la innata y permanente necesidad del ser humano de buscar, obtener, integrar, manejar y analizar información espacial para el desempeño de actividades básicas en su vida cotidiana tales como las relaciones familiares y sociales, prácticas laborales, religiosas, educativas, de compra, ocio, etc. que implican desplazamientos, etc. Se trata, pues, de una disposición primigenia que se expresa como interés y demanda de localizar personas, cosas, eventos, 
lugares, etc. para ligar dicha información con la estructura de necesidades del individuo.

b) Por otro, la notable (aunque no universal) propensión e interés de quienes poseen recursos o información (incluso la de su simple existencia localizada) con utilidad potencial para terceros, de darlos a conocer, por cuanto suponen soluciones a demandas latentes de estos, con lo que se convertirían en satisfactores de necesidades o deseos de otros, que les reportarían, a su vez, utilidades bajo diferentes formas. Ello originaría una corriente de información, georreferenciada de alguna forma, hacia los potenciales destinatarios («mercados»), buscando fomentar los intercambios de utilidades lucrativas, como las relaciones económicas por ejemplo, pero también no lucrativas, aunque no menos gratificantes, tales como las familiares, de amistad o sociales sensu lato.

Junto a los anteriores conceptos, querríamos proponer otro nuevo, relacionado con ellos, pero con una entidad algo distinta, lo cual justifica el ejercicio de su identificación y caracterización inicial aquí. Se trata del entorno personal próximo (EPP) como un ámbito dinámico de relaciones e interacciones espaciales potenciales para cada individuo, que le acompaña en su trayectoria espacio-temporal cotidiana, conteniendo tanto oportunidades, como amenazas.

La naturaleza del EPP, es decir, su contenido significativo para el individuo, vendría dada por elementos o componentes diversos que esbozamos a continuación.

A. Acceso: lugares u oportunidades a los que la persona puede llegar, desde donde se encuentra en cada momento, con escaso o irrelevante coste y nimia alteración de su plan de actividad momentáneo (i.e. aquí y ahora). La FIGURA 2 ilustra la ubicación del individuo, definida sobre anclajes como el domicilio o el lugar de trabajo, y unos potenciales destinos-oportunidades del entorno inmediato englobados dentro del EPP. En todo caso, habiendo asumido que los movimientos derivables conllevarían un coste o esfuerzo insignificante, cabe presumir una elevada probabilidad de ocurrencia de las mencionadas interacciones. Es patente la vinculación de este componente con el conocido concepto de accesibilidad espacial, en tanto que posibilidad de desplazarse desde un lugar a otros destinos (instalaciones, equipamientos, etc.). Particularmente está próximo a la idea que, en el enfoque de la «Geografía del tiempo», describía para un individuo dado su trayectoria espacio-temporal (pathline) y los sitios que desde cada lugar de cierta permanencia le eran alcanzables. Ello se plasmaba en los llamados prismas espacio- 
temporales que traducían el alcance de los desplazamientos factibles desde allí (vid. por ejemplo LENNTORP, 1976; CARLSTEIN et al. 1978; MILLER, 1991; MCBRIDE et al. 2002). La principal diferencia conceptual con nuestra definición radica en que, en lugar del ámbito alcanzable, el EPP se entiende como constreñido por la distancia accesible con un coste despreciable (a definir operativamente) y no con el coste máximo soportable. Así mismo nuestro concepto se relaciona con la idea topológica de «proximidad», cuya definición y trascripción métrica ha sido objeto de cierta atención (vid. YAO y THILL, 2005).

B. Visibilidad: zona que el individuo puede avistar con discernimiento suficiente como para apreciar e internalizar contenidos positivos o negativos relevantes en ella. Podrían mencionarse, a título de ejemplos conocidos, el dominio visible efectivo de la publicidad estática o el área de vigilancia eficaz desde un punto observatorio. La FIGURA 3 ilustraría el EPP visible de un posible visitante-turista sito en el centro de una plaza pública.

Figura 2. El entorno personal próximo (EPP) centrado sobre dos lugares comunes de la vida cotidiana y destinos de interacción potencial (puntos)

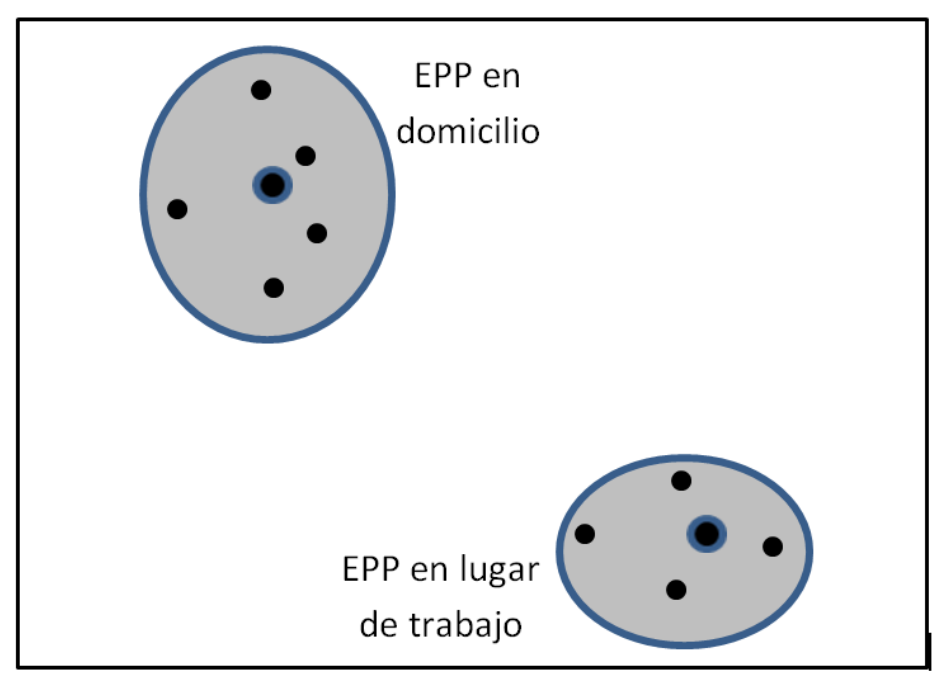

FUENTE.: Elaboración de Antonio Moreno

Conceptualmente y como criterio de demarcación del EPP cabría proponer que, para un individuo concreto, integraría el conjunto de lugares y ambientes con los que, dada la ubicación espacio-temporal de dicho 
individuo, sería factible y probable que este desarrollase algún tipo de relación (e.g. desplazamiento o flujo) con tales lugares y los entes allí asentados, y de acuerdo con las maneras regulares de operación espacial de dichos entes o fenómenos. La frontera o límite inferior del EPP coincidiría con la propia piel del individuo y el superior sería más variable, dependiendo del alcance espacial efectivo de cada tipo de interacción. Por ejemplo, para desplazamientos a ciertos comercios o servicios de proximidad podría situarse en muy pocos cientos de metros. Imaginaria y simplificadamente podríamos asimilarlo a un círculo o polígono (2D) o una semiesfera (3D) que «se desplaza» con el individuo. De una manera algo más realista cabría pensar en un dominio de forma irregular, de tamaño variable y dependiente, en su concreción, del tipo de movimiento o relación considerada, que delinearía una envolvente a cierta distancia de los lugares por donde discurre la vida cotidiana de la persona (vid. FIGURA 4). Igualmente, y recordando la primera ley de la Geografía de Tobler, parece razonable imaginar dicho EPP como afectado, en alto grado, por el fenómeno de descenso con la distancia, más que definido por un límite discreto y abrupto; ello conllevaría, ocasionalmente, unos límites exteriores borrosos.

Figura 3. Ejemplo de EPP según el criterio del espacio intraurbano visible (visualscape) desde la posición del punto

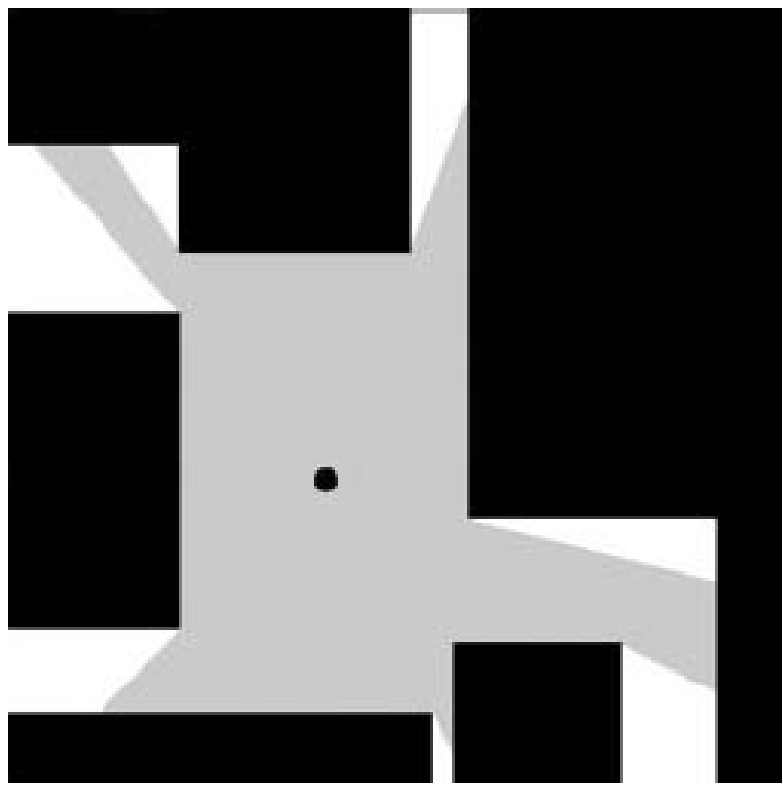

FUENTE.: LLOBERA (2003). 
Figura 4. El entorno personal próximo (EPP) como zona envolvente del conjunto de los lugares de actividad del individuo, definiendo un derredor de interacción potencial preferente para el mismo

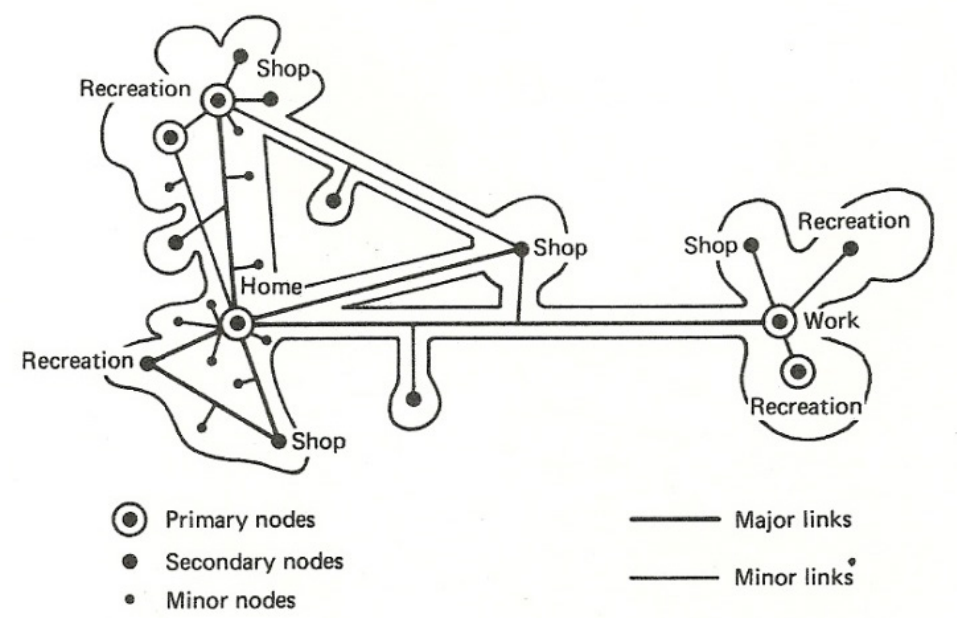

FUENTE.: GOLLEDGE (1978: 80)

C. Desde un ángulo distinto, cabría proponer que intervienen y cobran sentido en el EPP aquellas áreas de influencia, atracción o impacto de actividades o agentes dentro de las que se ubica o se mueve el individuo y que, por lo tanto, le pueden aportar, potencial o efectivamente, utilidades o desutilidades, incluso sin mediar aceptación o consciencia por parte de dicho individuo. En esa línea cabría señalar concretamente las amenazas sobrevenidas desde fuentes fijas o móviles que están lo bastante cerca como para poder impactar significativamente en la persona. Comprendería, por ejemplo, emisiones contaminantes de ruido, radiaciones, gases, partículas, etc. generadas por una actividad y que poseen un alcance espacial que «cubren» al individuo. Con ellas se relacionarían las molestias o perjuicios generados por esa actividad entre los afectables (i. e. dentro del área de alcance de la afección). Otros ejemplos concernirían a posibles peligros por explosiones, accidentes, incendios, etc. ocasionados por causas naturales o humanas (e. g. actividades productivas) o las contingencias provenientes de terceras personas, tales como contagio de enfermedades, amenazas a la integridad o a los bienes personales, etc. (vid. FIGURAS 5 y 6 ). Igualmente, podría considerarse como componente relevante las actuaciones que, desde la perspectiva de la competencia espacial, desarrollan los establecimientos comerciales o de servicios a la 
población sobre los consumidores «cercanos» por cuanto esa proximidad les facultaría para atraer al individuo-consumidor e intentar materializar una transacción económica, por ejemplo. En la medida en que la ubicación del individuo, instantánea o durante un cierto período, se sitúa dentro del área de atracción de un comercio, por ejemplo, podría ser susceptible de acciones de captación (por ejemplo, a través de mensajes o estímulos informativos).

Figura 5. Modelo de exposición espacio-temporal de la linea de un individuo a un prisma (izqda.) o a un vector (dcha.) de amenazas para su salud.

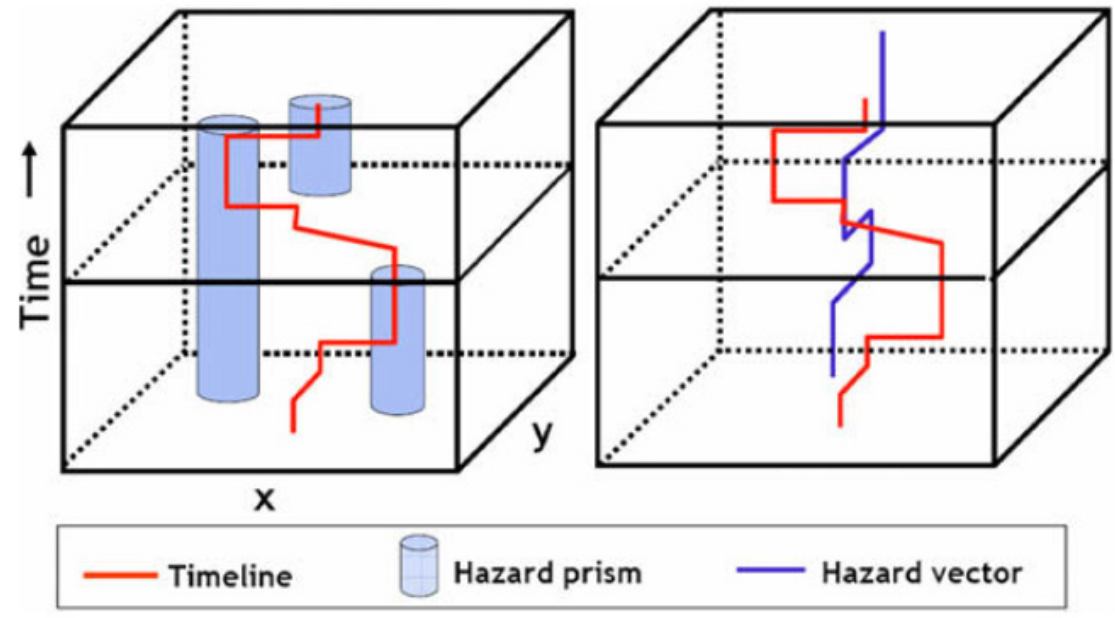

FUENTE.: SABEL et al. (2009)

Convendría recordar aquí, aunque sea de pasada, la relación que muchos de estos contenidos poseen con el concepto de externalidad espacial (vid. MORENO, 1995), por cuanto a menudo se provocan utilidades o desutilidades no compensadas económicamente entre emisor/fuente y receptor/afectado.

A efectos de análisis y de manejo, cabría proponer que el EPP se podría establecer y delimitar empíricamente, bien a partir de una multiplicidad de componentes o bien de alguno de ellos en particular. La decisión al respecto vendría dada por diversas consideraciones, tales como el tipo de fenómeno o práctica socio-espacial contemplada y el tipo de individuo. Por ejemplo, el EPP significativo de un adulto sito en su hogar o el de un turista sito en el centro urbano habría de ser parcialmente diferente, es decir, los componentes geoinformativos relevantes para cada uno de esos EPP serían desiguales, en atención a los intereses y prácticas factibles por cada una de esas personas en tales lugares y momentos. 
Figura 6. Lineas o trayectorias espacio-temporales de cuatro individuos, mostrando su inclusión en dos zonas distintas de peligros para la salud

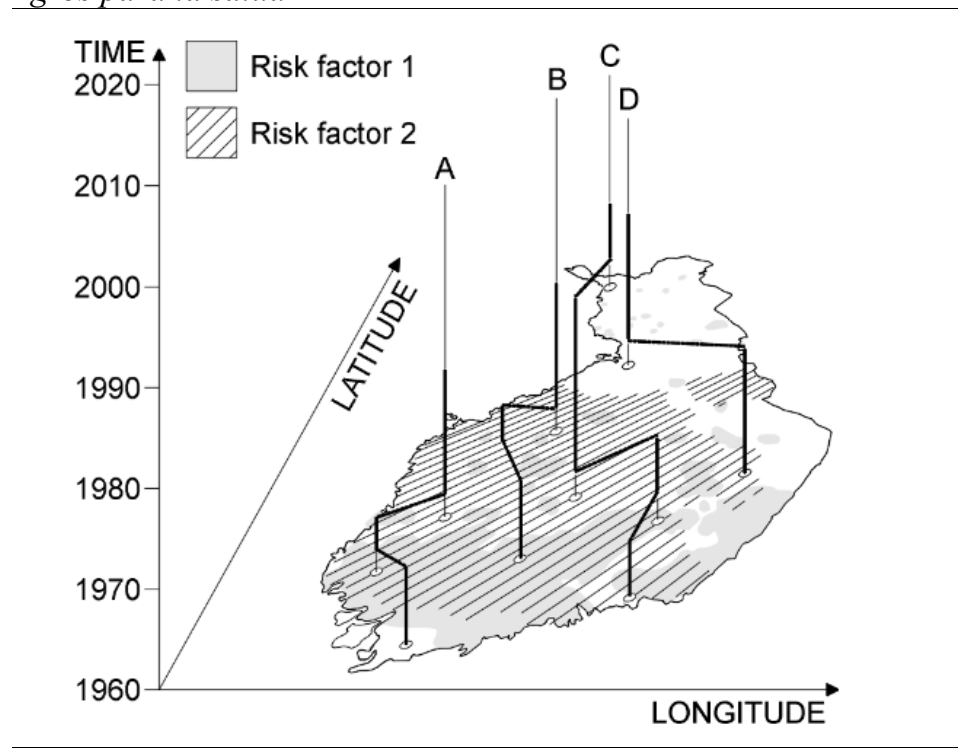

FUENTE.: LÖYTÖNEN (1998)

De la exposición anterior se quiere concluir que el EPP, pese a su carácter de constructo conceptual, posee una materialidad potencial, es decir, una naturaleza sustentada y definible en términos de interacción dinámica, esto es, móvil y contingente, como el individuo al que envuelve. Su importancia radica en que la persona lo gestiona rutinariamente en su decurso y despliegue cotidiano, por cuanto le reporta ventajas y contrariedades. Se constituye así en el dominio de intercambio potencial de utilidades entre la persona y el espacio circundante, por lo que cabe atribuir a tal derredor un interés preferente por parte de la persona. En una perspectiva diacrónica, esas interacciones potenciales podrían cursar de tres formas: a) ejecutarse conscientemente (e. g. dar lugar a un desplazamiento para examinar de cerca un elemento atisbado del paisaje); b) realizarse inconscientemente, i.e. sin que el individuo se aperciba de ellas (como por ejemplo respirar aire malsano); o c) no realizarse (e. g. por desconocimiento mutuo entre los agentes implicados, emisor y receptor (por ejemplo, no comprar en un establecimiento por ignorancia de su existencia). 


\section{LA SOCIEDAD DE LA GEOINFORMACIÓN (SGI) COMO MARCO NUEVO DE LA EXISTENCIA PERSONAL}

La SGI actualmente en gestación implica, como rasgo definitorio, que la GI adquiere un carácter tan constitutivo y esencial de su funcionamiento cotidiano, que sería impensable su ausencia (en cantidad, calidad, forma de presentación y acceso), por cuanto podría ocasionar un impacto funcional severo en la sociedad, o incluso su potencial colapso.

Como es bien sabido, históricamente la GI resultó limitada en cantidad y calidad, de difícil disponibilidad y de obtención costosa, de actualidad exigua, con desigual exactitud y, por tanto, plagada de incertidumbre. Por ejemplo, la mayoría de los mapas, soporte conspicuo de la GI, eran bienes algo sofisticados y su manejo estaba relativamente confinado a personas con un cierto nivel cultural y a especialistas. La GI al alcance del ciudadano corriente era parca y la vida ordinaria se nutría pobremente de ella. Se podría decir que muchas personas estaban en una situación de indigencia informativa sobre la espacialidad de los entes de su interés, tales como amigos, familiares, lugares, bienes, etc., lo que limitaba fuertemente su campo de información espacial. Por derivación, ello mermaba de manera drástica el ámbito de desarrollo de las actividades del individuo, i.e. su campo de actividad.

El advenimiento del estadio que llamamos SGI está suponiendo un cambio radical de escenario en el que dos elementos nuevos resultan determinantes: por un lado, la geotecnosfera digital y, por otro, la geoinformación digital. Ambos son los pilares que soportan la génesis del espacio digital o ciberespacio. La geotecnosfera actual (BUZAI y RUIZ, 2012), construida mediante la electrónica, la informática, la telemática y un sinfín de dispositivos, ha viabilizado la digitalización de la geoinformación, la cual está creciendo y circulando de manera formidable. Sobre esas bases ha cristalizado el llamado espacio digital o ciberespacio, cuya realidad descansa sobre la interconexión (mediante ordenadores, redes de cable o inalámbricas y aparatos varios) de personas y de lugares geolocalizados. Aunque se han propuesto diversas definiciones del mismo, la de MAYER et al. (2014) resulta conveniente:

"Cyberspace is a global and dynamic domain (subject to constant change) characterized by the combined use of electrons and electromagnetic spectrum, whose purpose is to create, store, modify, exchange, share and extract, use, eliminate information and disrupt physical resources».

Las propiedades de los flujos en un espacio tal, entre ellas la extraordinaria velocidad de respuesta a las búsquedas, están posibilitando conocer y compartir de forma más amplia, profunda y actualizada el conocimiento de la realidad 
geográfica entre quienes estén conectados a esas redes. En efecto, ahora observamos:

A. Que la información de los objetos, entes y eventos de interés es abundante, asequible y barata, obtenible cómodamente (a través de variados dispositivos móviles como iPad, teléfonos celulares, tabletas, ...), bastante exacta y, con frecuencia, actualizada. La información sobre los lugares fluye a raudales y bajo demanda, casi instantáneamente, con enorme facilidad, a la escala deseable, con legibilidad y flexibilidad notables y con contenidos elegibles (hasta cierto punto), merced a portales y servicios de geoinformación públicos o de empresas privadas.

B. Desde la perspectiva geográfica cabe subrayar, por un lado, que la localización de los datos puede ser establecida cualitativamente como información literal o verbal (estoy en...; tal evento sucede en...; podemos reunirnos en...) o cuantitativamente (y con transcripción cartográfica inmediata) a través de operaciones de georreferenciación automática de acceso universal, que permiten mostrar la distribución espacial de los fenómenos. Y por otro, que las antaño prestaciones casi exclusivas de sofisticados programas SIG corriendo en computadores, que permitían responder a preguntas como ¿dónde hay o está...? ¿cómo puedo ir a... y a través del medio de transporte...? ¿a qué distancia o tiempo puedo encontrar...? ahora están disponibles para cualquier ciudadano que posea un teléfono «inteligente» y de precio asequible.

La emersión de ese espacio digital está ocasionando un cierto número de consecuencias generales atingentes al funcionamiento espacial de las sociedades, de las cuales subrayaremos algunas, por su interés a los fines de este trabajo.

En primer lugar, el CIE del individuo se puede ampliar potencialmente lo indecible hasta abarcar, como límite imaginario, al conjunto del ciberespacio «abierto» (i.e. sin restricción de acceso para la persona). Además, incrementarlo resulta muy fácil, mediante consultas eficaces en tiempo real a través de los terminales y redes. Resolver una duda o subsanar una ignorancia sobre los lugares y sus atributos puede ser, en muchos casos, cuestión de segundos y a un coste nimio. Los límites al CIE vienen dados ahora, más que por la oferta o accesibilidad a la información, por la curiosidad (y su priorización) por parte de los individuos u organizaciones y la habilidad de búsqueda del demandante.

En segundo lugar, las telecomunicaciones han superado muchas de las limitaciones inherentes a la localización física personal y han abierto la puerta a una ubicuidad virtual; la multi-presencialidad está dejando de ser una utopía, 
pues una persona puede «estar presente digitalmente» en muchos sitios a la vez, no solo ya mediante el patrón de relaciones de uno a muchos, como en las tradicionales emisiones vía radio o TV, sino mediante la multi-interacción simultánea entre varios agentes (a través de flujos telemáticos bidireccionales en las redes digitales). Ello implica un ingente aumento del margen de libertad individual, en particular a la hora de elegir qué hacer, dónde, cuándo, cómo, con quién, etc.

En tercer lugar, procede recordar que la presencia y actos de los agentes operando en el espacio digital deja rastro, es decir, la información del actor y de sus acciones queda registrada a través de sistemas y dispositivos varios, abriendo unas inmensas posibilidades de conocer conductas y rasgos de las personas y las organizaciones $\mathrm{y}$, por derivación, a unas potencialidades de aprovechamiento útil por otros. Los individuos y los objetos existen en el ciberespacio, por cuanto cuentan con códigos de identificación (e.g. los de los terminales de acceso al ciberespacio), por los cuales resultan «visibles» y pueden ser localizados, rastreados en su posición y a través de la huella de los movimientos que realicen, tanto en el espacio geográfico, como en el digital (vid. ISAACSON y SHOVAL, 2006). Baste recordar que, por ejemplo, cada dispositivo móvil o celular posee tales códigos de identidad (y los de su propietario o usuario / portador), al igual que el conocido DOI para los documentos digitales publicados en Internet.

Finalmente, y en virtud de esa presencia virtual, el agente (individual o corporativo) puede ser destinatario y objeto de acciones, deseadas o no por él, y decididas por terceros, desde las más amistosas o beneficiosas hasta las hostiles y peligrosas. Como sucede en el espacio físico, la trasposición digital del individuo está sometida al amplio abanico de interacciones que en aquel espacio existen, desde las de carácter abierto, hasta las que atañen a su privacidad, con la particularidad de que el material manejado es la información digital, la cual puede ser utilizada con fines muy dispares. Son bien sabidas las implicaciones de extraordinario calado que todo ello tiene en materia de seguridad, imagen, etc. y que no consideraremos aquí, por no concernir al interés primordial de este trabajo.

\section{ALGUNAS CONSECUENCIAS DE LA SGI EN EL CONO- CIMIENTO Y CONDUCTA ESPACIAL DEL CIUDADANO}

Querríamos empezar este apartado con una anécdota real, expresiva de ciertas conductas espaciales y posibilidades en nuestra geotecnosfera, y que sirve de ilustrativo contrapunto respecto a momentos pretéritos. 
Durante un viaje de ocio visitando en automóvil varios municipios de una comarca española, uno de los viajeros extravió un objeto de importante valor personal, hecho del que se percató al día siguiente, cuando se había alejado bastante en la zona. La eventual recuperación del bien se vislumbraba muy incierta debido al desconocimiento de dónde se produjo el extravio, y a que deshacer el camino indagando presencialmente en los diversos lugares y establecimientos visitados no compensaba el precio del bien. Como estrategia alternativa se realizó, en primer lugar, un listado de los sitios probables (establecimientos principalmente) donde pudo olvidarse el artículo, lo cual redujo bastante el espacio de búsqueda. De cara a acotar más las alternativas a rastrear, se recurrió a un smartphone cuyos servicios de cartografía y de visualización de imágenes digitales del viario urbano permitieron identificar los establecimientos visitados, cuyos nombres incluso se ignoraban. Seguidamente, y usando las prestaciones búsqueda por Internet con el mencionado smartphone se pudo averiguar la dirección postal y el teléfono de los establecimientos «candidatos» más probables. Unas pocas llamadas realizadas con el mismo dispositivo bastaron para localizar exactamente el paradero del bien perdido y concertar una cita para la recogida. El trayecto a realizar para ese fin pudo optimizarse mediante el apoyo de un navegador GPS en el automóvil, maximizando la velocidad y evitando las infracciones en tramos controlados por radares de tráfico. Conseguida la recuperación del objeto, fue posible incluso llevar a cabo algunas visitas adicionales en la zona, merced al tiempo ahorrado y al buen ánimo por el feliz desenlace. Sin lugar a dudas, hasta hace unos pocos años el artículo habría sido considerado como definitivamente perdido.

Hablando ya en términos generales, en la SGI el acceso el acceso abierto al ciberespacio aporta todo un universo de posibilidades para satisfacer más y mejor las necesidades y deseos del ciudadano. Centremos nuestra atención en aquéllas que, desde el punto de vista conductual, involucran un desplazamiento físico. El proceso simplificado del ser humano podría describirse en estos términos:

Situación de estímulo o deseo personal $\rightarrow$ búsqueda de información espacial relevante y análisis de la misma $\rightarrow$ decisión (dónde, cuándo, cómo) $\rightarrow$ desplazamiento al destino y ejecución de la actividad $\rightarrow v a-$ loración retrospectiva de la experiencia (éxito o fracaso).

El anterior esquema resulta compatible con otros enunciados en la bibliografía, por ejemplo el de DOWNS (1970) y, como ha quedado evidenciado en ella, en varios momentos de esa secuencia la geoinformación juega un papel 
crítico acerca de la conveniencia, viabilidad, costes, éxito, etc. del plan imaginado por el individuo.

La lógica asumida aquí sobre la conducta personal, en la doble dimensión del espacio físico y digital, sería como sigue (FIGURA 7): en una situación hipotética (espacio-temporal) del espacio de actividad la persona, y ante un impulso propio que le lleva a buscar geoinformación o por la detección de su presencia por otro agente del ciberespacio (que le proporciona datos estimulantes), se concreta un EPP enriquecido y aumentado por esa nueva información añadida, el cual actúa como base para sus decisiones comportamentales inmediatas. Éstas, por tanto, responden a un contexto nuevo en el que la inmediatez del acceso a esa nueva información cambia el espacio conocido, ampliando el conjunto de destinos elegibles (spatial choice set) y puede propiciar flujos distintos a los previsibles en la situación inicial. De nuevo, en el momento de la realización del desplazamiento y de la actividad, la disponibilidad de acceso al ciberespacio abre la opción de consultas simultáneas (para ampliación o comprobación de datos, por ejemplo). La movilidad virtual, informativamente hablando, por ese cosmos digital se configura así en un asistente casi permanente y en tiempo real del individuo.

Recordemos, por ejemplo, la práctica ya habitual de averiguar por el ciudadano qué establecimientos ejerciendo ciertas actividades le quedan cerca (e. g. en su CAE y EPP) o, de otro lado, y pensando ahora en la seguridad (¿sostenibilidad?) individual si en el lugar en que se está o se piensa recorrer concurre alguna amenaza (e. g. contaminación alta) o circunstancia adversa (concentración de accidentes o de delitos) que permita reconsiderar la decisión o el itinerario.

El hecho diferencial en nuestro tiempo estriba en que el acceso al ciberespacio está contribuyendo decisivamente a despejar incertidumbres en las diversas facetas de un plan cualquiera de movilidad en ciernes del individuo. En efecto, la disponibilidad allí de una mayor y más exacta geoinformación posibilita avistar decisiones espaciales mejor fundadas, más realistas, más eficientes (i.e. con menos costes y errores) y más efectivas (menos fracasos). Parece plausible concluir que esa ayuda geoinformativa digital desemboque en una experiencia gratificante y que, por ende, se valore de manera positiva y creciente el recurso al ciberespacio. De ello cabe inferir algunas consecuencias para el individuo.

Un primer efecto radica en que éste se plantee y adopte ya una conducta espacial permanentemente asistida por dichos recursos, que pasan así a integrarse en la cotidianidad humana. Podría hablarse por lo tanto de una situación de dependencia de ese cosmos digital, similar a la que hemos experimentado 
históricamente respecto a muchas máquinas con las que se ha establecido una relación finalmente rutinaria, pero que cambió el modo de vida previo.

Figura 7. A) El campo de actividad espacial (CAE como hexágono) y el entorno espacial próximo (EPP) inicial, alrededor de la localización del individuo; B) El ciberespacio como recurso para enriquecer el campo de información espacial (CIE) del individuo; C) El EPP modificado, tras la ganancia informativa obtenida del ciberespacio, el CAE ampliado y los desplazamientos desencadenados (flecha) hacia destinos identificados e integrados en el conjunto elegible

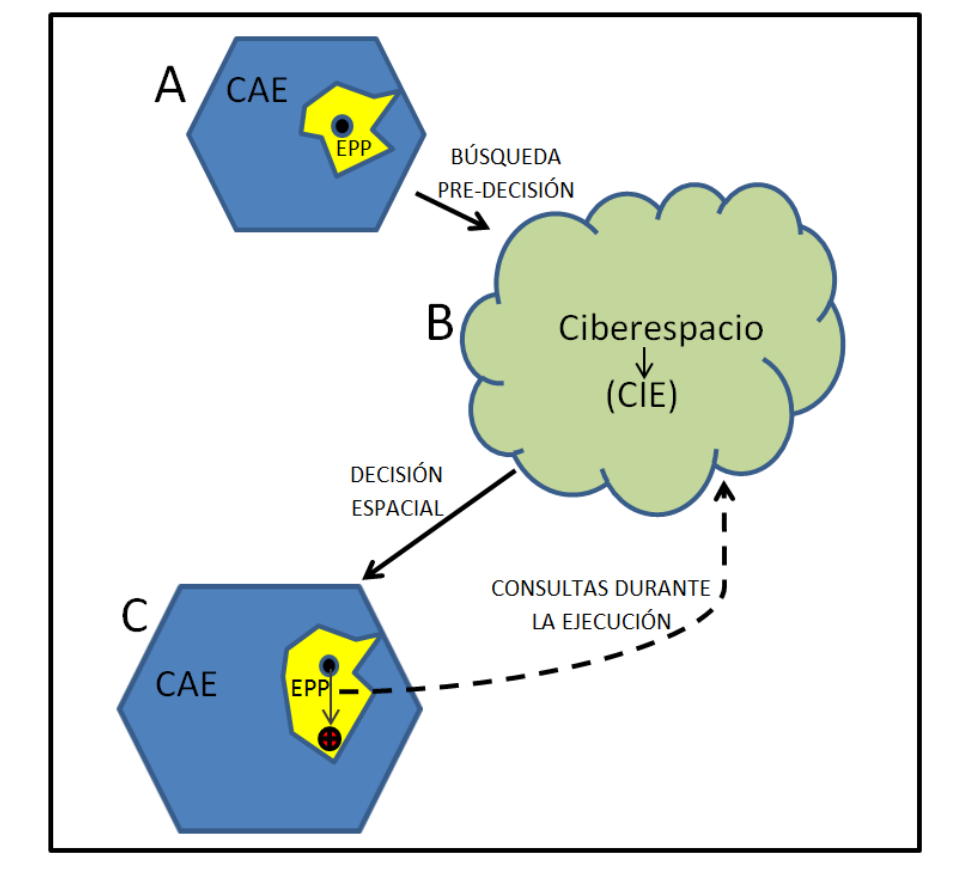

FUENTE.: Elaboración de Antonio Moreno

Una segunda consecuencia observable estriba en la propensión a una hiperactividad del ser humano. En efecto, la capacidad de recabar más información (incluso en tiempo real), que contiene estímulos para el individuo, y la constatación de una mayor eficiencia (o productividad) en la ejecución de actividades y de efectividad (éxito de las decisiones y sus resultados) parece estar conduciendo a una implicación de las personas en más actividades, por resultarles ya viables y satisfactorias. Si la generalización del automóvil, por ejemplo, cambió los hábitos y la movilidad espacial, ahora estaríamos ante un nuevo salto adelante: frente al tempo pre-electrónico o informacional, más 
pausado, en el que los desplazamientos se veían constreñidos, no tanto por la capacidad de los medios de transporte mecánico, como por la limitada información sobre los destinos alcanzables, ahora nos hallamos con la importante novedad de que el conocimiento de las oportunidades existentes en muchos lugares (y momentos) es conseguible de manera ágil a través de búsquedas rápidas en dispositivos móviles. Es decir, la cuestión de si es factible realizar tal actividad y ahora en ciertos sitios puede responderse con alto grado de certeza, permitiendo por tanto una formación de decisiones personales o grupales (implicando desplazamientos) mucho más atinada y una consecuente realización de las mismas. Cabe por ende apuntar a un salto cuantitativo notable en la densidad o ritmo temporal de flujos personales y de las interacciones, conformando un nuevo tempo o ritmo vital en la SGI.

Procede hipotetizar además, que el proceso entrañaría una relación, en gran medida, de tipo circular: una mayor implicación en actividades, permite aumentar el CIE, lo cual a su vez generaría nuevos estímulos y motivaciones para interactuar, participar y desplazarse tanto en el CAE, como en el EPP.

En resumen, el CIE de cada persona crece de forma gigantesca (y a escala global) y ello está cambiando radicalmente las bases y el contexto de la acción personal. El mapa mental del espacio de interés individual deviene más denso, esto es, más cargado de lugares y entidades conocidos y, por tanto, con significado para el sujeto. La ignorancia sobre terceros vivencialmente cercanos, como los amigos o la familia (por ejemplo sobre su situación en cada momento), impidió históricamente imaginar y desplegar iniciativas y atender a deseos y aspiraciones latentes. El enriquecimiento de la información espacial del individuo respecto a los otros fomenta una creciente interacción entre sujetos que toma formas diversas (vid. Kellerman, 2012 a y b). Por un lado aumentan las relaciones virtuales en el espacio digital (flujos de información). Es constatable el extraordinario éxito de las redes sociales y de otras manifestaciones soportadas en ese medio. La movilidad virtual se ha disparado y, junto a flujos informativos diversos, también los de carácter geodigital están expandiéndose notoriamente, al aportar las utilidades de la dimensión espacial de la existencia humana. Pero también está siendo espoleada la movilidad física habitual, sea terrestre, marítima o aérea, por las razones antes esgrimidas. El campo de actividad personal (CAE) se modifica en dos sentidos: en intensidad y en extensión. Cabe, en fin, postular que la geotecnosfera actual, combinando superiores medios de transporte y un riquísimo ciberespacio (informativamente hablando), catalizaría más movimientos en el entorno de interés individual (el EPP) y a la par permite una ampliación del mismo. 


\section{LA IDENTIFICACIÓN DE OPORTUNIDADES EN LAS NUEVAS PRÁCTICAS ESPACIALES DE LOS CIUDADANOS COMO RETO PARA LA GEOGRAFÍA EN LA SGI}

Como hemos argüido anteriormente, el advenimiento de la SGI genera una nueva situación existencial para la persona que, desde el punto de vista geográfico, se plasma en potencialidades y desafíos a los cuales deberíamos atender como colectivo que busca proporcionar soluciones o respuestas espaciales a las necesidades de la sociedad. Plantear ese objetivo supone revisar nuestro papel como productores de información elaborada (saber geográfico y geodatos) que sea relevante para los destinatarios en el contexto de la geotecnosfera actual y de su futura evolución.

Como es sabido, información georreferenciada de diverso tenor está ya disponible en el ciberespacio y está siendo consultada por los ciudadanos de manera habitual a la hora de tomar decisiones sobre movilidad espacial y en el decurso del desplazamiento. Recuérdese por ejemplo, la previsión meteorológica sobre lugares, los rasgos de lugares (para hipotéticas visitas), los atributos de recursos (superficies, capacidades, atractivos, etc.), la identificación de establecimientos (e. g. de servicios o comerciales) cercanos a la ubicación del usuario, las rutas (distancia, tiempo, descripción del itinerario) para ir a lugares, etc.

En este contexto, dejando de lado a las organizaciones de cualquier tipo y considerando exclusivamente a los ciudadanos individuales como destinatarios y consumidores finales de geoinformación, un foco de nuestro pensamiento estratégico debería recaer en la identificación de prácticas y usos sociales, cuya ejecución o despliegue espacial puedan ser susceptibles de mejora en su eficiencia o efectividad espacial, entendida ésta no solo como referida al espacio físico, sino también al digital. Más concretamente se trataría de, por un lado, dilucidar qué contenidos geoinformativos, disponibles o proporcionables desde el ciberespacio, podrían resultar de utilidad suficiente, por conectar con necesidades y deseos de los ciudadanos en un lugar y momento concretos, como para justificar una acción positiva de provisión en ese sentido por parte de aquéllos agentes (individuales o colectivos, como las organizaciones) que generan dichos contenidos de geoinformación. El objetivo principal radicaría, por tanto, en detectar oportunidades de poner en valor información georreferenciada (recursos, problemas, oportunidades, etc.) para los individuos. Se trata de una labor semejante a la que en las empresas innovadoras se lleva a cabo para detectar nuevas líneas de negocio y de desarrollo.

Por otro lado, y dentro de ese análisis estratégico, se habría de indagar (identificar y evaluar) los grupos de población o segmentos de mercado (usando 
la terminología del marketing) susceptibles de implicarse en dichas prácticas. Su dimensionamiento y caracterización podrían facilitar la respuesta acerca de la «rentabilidad» de dicha geoinformación, lo que implicaría avistar a una amplitud suficiente del colectivo de destinatarios potenciales. Parece obvio establecer que, en una sociedad atenta tanto a los principios de competitividad espacial, como a los de justicia socio-espacial, dicha rentabilidad debería ser entendida no solo en términos de beneficio económico, sino también en términos de beneficio o bienestar social, es decir, no lucrativo.

Una apostilla adicional cabría señalar acerca del caso particular de los grupos demográficos vulnerables (menores de edad, ancianos, mujeres, discapacitados, etc.) cuyas limitaciones parece que les habrían de hacer acreedores de un especial cuidado. En efecto, el acceso ilimitado al ciberespacio o la «invasión» por agentes terceros les puede exponer a oportunidades singulares (y en muchos casos deseables), pero también a peligros superiores por su debilidad. En estos casos, la probabilidad de ocurrencia de un hecho indeseable podría ser avisada (por ejemplo, si la amenaza cae en su EPP) o reducida (por ejemplo, bloqueando la geoinformación de destinos atractivos, pero nocivos en su CIE).

Un aspecto más de ese reto, concerniría a los procesos y mecanismos de la distribución de esa geoinformación digital: cómo (canal), dónde, cuándo, etc. Al respecto procede traer a colación aquí, como conceptos especialmente relevantes, los mencionados antes de campo de actividad espacial, campo de información espacial y entorno personal próximo. En efecto, asumiendo que la incorporación de un individuo al ciberespacio, a través de la conectividad otorgada por la geotecnosfera, abre las puertas al vastísimo stock de datos presente en aquél, el acceso a la geoinformación relevante para dicha persona se ve condicionado por factores varios, tales como la forma de distribución de la geoinformación, la usabilidad de la misma, la destreza del individuo para buscarla y asimilarla, etc.

El reto podría multiplicarse si, como está sucediendo, nos preguntamos por demandas latentes de segmentos concretos de la población. Aunque la estrategia de ciertos proveedores busca el negocio y por tanto prioriza servicios de esta índole centrados en contenidos estándar y de uso masivo (por ejemplo, el conocido asistente personal conocido como Google Now, lanzado en 2012), hay margen para desarrollar servicios de geoinformación digital para segmentos de destinatarios más minoritarios (grupos sociales afines por algún interés compartido, e. g. alguna afición o necesidad particular). Se trataría de unas aplicaciones próximas al concepto de micro-marketing, por ser factible, llegado el caso, el ajuste a tipos de personas distintos. 
Está ya constatado en experiencias reales que las organizaciones privadas que ofrecen bienes y servicios pueden obtener ventajas competitivas desarrollando desde el enfoque descrito servicios de geoinformación (e.g. tipo location-based services) al consumidor y que las expectativas de negocio que se vislumbran en ese campo son muy prometedoras, según se desprende de estudios de prospectiva como el realizado por Oxera Consulting (2013) para Google. Adicionalmente, y desde la perspectiva pública, la atención a ese nuevo proceso de gestación de decisiones espaciales debería conducir al impulso de actuaciones más directas hacia el ciudadano (usuario final) en esa misma línea. Ellas podrían ser muy variadas, desde las inspiradas en lograr una superior competitividad territorial, a través de una mejor geoinformación, por ejemplo, a visitantes o turistas, hasta las destinadas a mejorar el bienestar de las personas, a través de aumentar el conocimiento de las oportunidades (e. g. recursos disponibles o amenidades accesibles) o de los peligros incluidos dentro del EPP.

\section{A MODO DE BREVE CONCLUSIÓN}

La sociedad de la geoinformación se nos aparece cada vez más como un escenario revolucionario por los cambios que entraña, en particular por la cantidad de nuevas oportunidades que están eclosionando y que se pueden poner en valor. Los ciudadanos con acceso al ciberespacio están teniendo una oferta de geoinformación que altera el marco de su existencia, incidiendo y transformando importantes realidades conceptuales que los geógrafos han identificado desde hace tiempo, como el campo de actividad y el de información espacial. Ellos, junto con el aquí introducido como EPP, aportan claves instrumentales para comprender la conducta espacial humana, por cuanto suponen el marco de la propia existencia. En este ensayo, altamente especulativo, se han señalado una serie de cambios, determinantes y procesos emergentes en este período histórico, que correspondería calificar sin ambages como SGI, poniendo especial atención en los impactos que están ocasionando en la praxis espacial de los ciudadanos.

Al respecto procede insistir en que el ciberespacio se está constituyendo en un asesor permanente antes, durante los desplazamientos y a lo largo de los períodos de realización de las actividades, facilitando la toma de decisiones y el replanteamiento de las mismas en tiempo de ejecución real. Ello aumenta la potencialidades para el individuo (en términos de libertad de elección y de reajustes espacio-temporales), a la par que refuerza la confianza en el proceso de formación y ejecución de decisiones espaciales. En resumen, cada vez más la existencia humana se está viendo infiltrada por los componentes de esta geotecnosfera, que tantas utilidades está reportando y de forma continuada. 
A partir de ello, se han esbozado algunos desafíos que se dibujan para la geografía en el horizonte actual, señalando las fases y momentos de la formación y ejecución de decisiones espaciales humanas en los que se recurre al ciberespacio como fuente de recursos informativos que ayuden en aquella labor que realizamos tan frecuentemente. En suma, se trata de plantear cuándo proporcionar geoinformación relevante para el individuo, que vaya al encuentro de sus necesidades básicas y rutinarias. Algunas experiencias descritas en THILL (2009) para el caso de los desplazamientos resultan bastante ilustrativas.

Aunque la cuestión de qué conocimiento geográfico y servicios ofertar para esos fines no se ha tratado sistemática y detalladamente aquí, sí se pueden vislumbrar a partir de los contenidos que, según se ha expuesto, conformarían el EPP, como ámbito inmediato y de prioritaria relevancia para el ciudadano. También queda pendiente de examinar en profundidad el importante punto relativo a la privacidad personal, puesto que, como es sabido, muchos de estos servicios prestados desde el ciberespacio implican disponer de información sobre el individuo (a través de su terminal) tales como su ubicación, su perfil, su comportamiento, etc. La aceptación personal previa parece un requisito imprescindible, por ejemplo, cuando se le propone descargar las conocidas APP en los smartphones y aceptar simultáneamente que accedan a información del dispositivo, mucha de la cual puede ser privada.

En cualquier caso, como productores de saber geográfico y de geoinformación elaborados, habríamos de reflexionar ampliamente sobre ese frente prioritario, pues ello ayudará a imaginar, concebir y diseñar qué contenidos podrían ser de más utilidad (por atender ciertas necesidades o deseos), para quiénes, bajo qué formato y cómo distribuirlo para que llegue eficazmente a los destinatarios potenciales en el momento y el lugar (i. e. el EPP) apropiados. Parece evidente que obrar así supondría una gran oportunidad de contribuir decisivamente a una renovada relevancia del conocimiento geográfico para la ciudadanía.

\section{BIBLIOGRAFÍA}

Amedeo, D.; Golledge, R. G.; Stimson; R. J. (2009): Person-Environment-Behavior Research: Investigating Activities and Experiences in Spaces and Environments. New York, Guilford Press.

BUZAI, G.; RUIZ, E. (2012): «Geotecnósfera. Tecnologías de la información geográfica en el contexto global del sistema mundo», Anekumene. 4, 88-106.

CAPEL, H. (1973): «Percepción del medio y comportamiento geográfico», Revista de Geografia, vol. VII., 58-150.

CARLSTEIN, T.; PARKES, D.; THRIFT, N. (1978): Human activity and time geography. Timing space and spacing time. Edward Arnold, Londres, v. 2. 
Downs, R. M. (1970): «Geographic space perception: past approaches and future prospects», Progress in Geography, vol. II. 65-108.

Golledge, R. (1978): «Learning about urban environments» en CARLSTEIN, T.; PARKES, D.; THRIFT, N. (Eds.): Making sense of time. Londres, Edward Arnold, 76-98.

GOLLEDGE, R.; STIMSON, R. (1997): Spatial behavior: a geographic perspective. New York, The Guilford Press.

ISAACSON, M.; SHOVAL, N. (2006): «Application of tracking technologies to the study of pedestrian spatial behavior», The Professional Geographer, 58 (2), 172-183.

JANELLE, D.; HoDGE, D. (2000): Information, place, and cyberspace: issues in accessibility. Berlín, etc. Springer.

Kellerman, A. (2012a). Daily spatial mobilities: physical and virtual. Farnham, Ashgate.

Kellerman, A. (2012b): «Potential mobilities», Mobilities, 7(1), 171-183.

LENNTORP, B. (1976): «Paths in space-time environments: A time-geographic study of the movement possibilities of individuals», Lund Studies in Geography, Series B (Human Geography), vol. 44.

LÖYTÖNEN, M. (1998): "GIS, time geography and health», en GATRELL, A. y LÖYTÖNEN, M. (eds.): GIS and health (GIS DATA VI). Londres, Taylor and Francis, 97-110.

LLOBERA, M. (2003): «Extending GIS-based visual analysis: the concept of visualscapes», International Journal of Geographical Information Science, 17(1), 25 48.

Mayer, M.; Martino, L.; Mazurier, P.; TzVetKova, G. (2014): How would you define Cyberspace? First Draft Pisa, 19.05.2014

https://www.academia.edu/7096442/How would you define Cyberspace

McBRIDE, S.; MA, D.; EsCOBAR, F. (2002): «Management and visualisation of spatiotemporal information in GIS», presented at SIRC 2002 - The 14th Annual Colloquium of the Spatial Information Research Centre, University of Otago, Dunedin, New Zealand, 13 p. http://www.geogra.uah.es/patxi/McBride02_Viz temp data.pdf

MiLlER, H. (1991): «Modelling accessibility using space-time prism concepts within geographical information systems», International Journal of Geographical Information Science, 5, 287-301.

MoRENO JimÉNEZ, A. (1995): «La medición de externalidades ambientales: un enfoque espacio-temporal», en Anales de Geografía de la Universidad Complutense, $15,485-496$.

http://revistas.ucm.es/index.php/AGUC/article/view/AGUC9595220485A

MORENO JIMÉNEZ, A. (2004): «Nuevas tecnologías de la información y revalorización del conocimiento geográfico», Scripta Nova, VII, 170 (62), 18 p. www.ub.es/geocrit/sn/sn-170.htm

MoRENO JiMÉNEZ, A. (2010): «GeoFocus: diez años en el camino hacia la sociedad de la geoinformación», GeoFocus (Editorial), 10, 1 - 6.

MORENO JiMÉNEZ, A. (2013): «Entendimiento y naturaleza de la cientificidad geotecnológica: una aproximación desde el pragmatismo epistemológico», Investigaciones Geográficas, 60, 5-36. 
http://rua.ua.es/dspace/bitstream/10045/34742/1/Investigaciones_Geograficas_6 $\underline{0 \text { 01.pdf }}$

Mumford, L. (1934). Technics and Civilization. Trad. Esp. Técnica y civilización. Madrid, Alianza Editorial (2006).

OXERA CONSULTING LTD. (2013). What is the economic impact of the Geo services. Prepared for Google. Oxford y Bruselas.

http://www.oxera.com/Oxera/media/Oxera/downloads/reports/What-is-theeconomic-impact-of-Geo-services 1.pdf

POTRYKOWSKI, M.; TAYLOR, Z. (1984): Geografia del transporte. Barcelona, Ariel.

SABel, C.E., Boyle, P., RAAB, G., LÖYTÖNEn, M.; MAasilta, P. (2009): «Modelling individual space-time exposure opportunities: A novel approach to unravelling the genetic or environment disease causation debate», Spatial and SpatioTemporal Epidemiology, 1, 85-94.

THILL, J. C. (2009, Ed.): Geographic information systems in transportation research. Emerald, 2 vols.

Timmermans, H. (2004): «Retail location and consumer spatial choice behavior», en Applied Geography. A World Perspective. Springer Netherlands. GeoJournal Library, Vol. 77, 133-147.

WiLson, M. I.; Kellerman, A.; COREY, K.E. (2013, Eds.): Global information society: technology, knowledge, and mobility. Lanham, etc. Rowman and Littlefield.

YAO, X.; THILL, J.-C. (2005): «How far is too far? - A statistical approach to contextcontingent proximity modeling», Transactions in GIS, 9 (2), 157-178. 